\title{
COMPUTATIONAL ELASTOCACOUSTICS OF UNCERTAIN COMPLEX SYSTEMS AND EXPERIMENTAL VALIDATION
}

\author{
C. Soize ${ }^{1}$, C. Chen ${ }^{1}$, J.-F. Durand ${ }^{1}$, D. Duhamel ${ }^{2}$, L. Gagliardini ${ }^{3}$ \\ ${ }^{1}$ Universite de Marne la Vallee, Laboratoire de Mecanique \\ 5 Bd Descartes, 77455 Marne la Vallee, France \\ e-mail: soize@univ-mlv.fr \\ ${ }^{2}$ Ecole des Ponts, Laboratoire Analyse des Materiaux et Identifications \\ 6-8 Avenue Blaise Pascal, 77455 Marne la Vallee, France \\ ${ }^{3}$ PSA Peugeot-Citroen \\ Route de Gisy, 78943 Velizy-Villacoublay Cedex, France
}

Keywords: Computational, Elastoacoustics, Uncertainties, Propability, Experiments

\begin{abstract}
The paper deals with the robustness of uncertain computational elastoacoustic models in low-and medium-frequency ranges. The elastoacoustic system is made up of a heterogeneous viscoelastic structure coupled with an internal acoustic cavity filled with a dissipative acoustic fluid. A reduced mean elastoacoustic model is deduced from the mean finite element model by using the modal approach with the structural modes of the structure and the acoustic modes of the acoustic cavity. Data uncertainties and model uncertainties are taken into account by using a nonparametric probabilistic approach for the structure, for the acoustic cavity and for the vibroacoustic coupling interface. The main objectives of this paper are (1) to present experimental validation of the nonparametric probabilistic approach of model uncertainties and to propose methods to perform the experimental identification of the probabilistic model parameters, (2) to analyze the robustness of computational elastoacoustic models with respect to model and data uncertainties, (3) to study uncertainty propagation through complex elastoacoustic systems. Two experimental configurations are analyzed with the stochastic computational elastoacoustic model. The first experimental configuration is made up of a composite sandwich panel coupled with an acoustic cavity constituted of a simple rigid box. Experimental measurements have been performed for 8 manufactured composite panels. The second experimental configuration is a car made up of a complex heterogeneous structure coupled with a complex acoustic cavity. Experimental measurements have been performed for 22 manufactured cars of the same type with optional extra.
\end{abstract}




\section{INTRODUCTION}

The paper is devoted to computational elastoacoustics in low- and medium-frequency ranges of uncertain complex systems made up of a viscoelastic heterogeneous structure coupled with an internal acoustic cavity filled with a dissipative acoustic fluid. Usually, data uncertainties are taken into account by using a parametric probabilistic approach allowing uncertain parameters of the computational model to be modeled by random variables. The mathematical-mechanical modeling process of the designed elastoacoustic system used to construct the computational model introduces model uncertainties which cannot be taken into account by the parametric probabilistic approach. Consequently, we propose to use the nonparametric probabilistic approach of data and model uncertainties recently introduced (see $[9,10])$. The main objectives of this paper are (1) to present experimental validation of the nonparametric probabilistic approach of model uncertainties and to propose methods to perform the experimental identification of the probabilistic model parameters, (2) to analyze the robustness of computational elastoacoustic models with respect to model and data uncertainties, (3) to study uncertainty propagation through complex elastoacoustic systems. Two experimental configurations are presented and analyzed. The first experimental configuration is made up of a composite sandwich panel (the structure) coupled with an acoustic cavity constituted of a simple rigid box. We are interested in the internal noise produced by the vibration of the structure induced by a point force applied to the structure. Experimental measurements have been performed for 8 manufactured composite panels (see $[1,2])$. The second experimental configuration is a car made up of a very complex viscoelastic heterogeneous structure coupled with a complex acoustic cavity. We are interested in the booming noise which is the internal noise produced by the vibration of the structure induced by engine vibrations. Experimental measurements have been performed for 22 manufactured cars of the same type with optional extra (see $[3,4])$.

\section{UNCERTAINTIES IN THE PREDICTIVE MODEL OF A REAL ELASTOACOUS- TIC SYSTEM}

The designed elastoacoustic system is the system conceived by the designers and analysts. A designed elastoacoustic system, made up of a structure coupled with an internal acoustic cavity, is defined by geometrical parameters, by the choice of materials and by many other parameters. A designed elastoacoustic system such as a car is a very complex elastoacoustic system. The real elastoacoustic system is a manufactured version of the system realized from the designed elastoacoustic system. Consequently, the real elastoacoustic system is a man-made-physical system which is never exactly known due to the variability induced for instance by the process. The objective of a predictive model is to predict the output $\left(\mathbf{v}^{\exp }, p^{\exp }\right)$ of the real elastoacoustic system to a given input $\mathbf{f}^{\text {exp }}$, in which $\mathbf{v}^{\exp }$ is the response in displacement of the structure and where $p^{\exp }$ is the acoustic pressure inside the acoustic cavity. Such predictive models are constructed by developing mathematical-mechanical model of the designed elastoacoustic system for a given input (see Figure 1). Consequently, the mean model has an input $\underline{\mathbf{f}}$ modeling $\mathbf{f}^{\text {exp }}$, an output $(\underline{\mathbf{v}}, \underline{p})$ modeling $\left(\mathbf{v}^{\exp }, p^{\exp }\right)$ and exhibits a vector-valued parameter $\underline{\mathbf{s}}$ for which data has to be given. The errors are related to the construction of an approximation $\left(\underline{\mathbf{v}}^{n}, \underline{p}^{n}\right)$ of the output $(\underline{\mathbf{v}}, \underline{p})$ of the mean model for given input $\underline{\mathbf{f}}$ and parameter $\underline{\mathbf{s}}$ and have to be reduced and controled using adapted methods developed in applied mathematics and in numerical analysis. The mathematical-mechanical modeling process of the designed elastoacoustic system introduces two fundamental types of uncertainties: data uncertainties and model uncertainties. Data uncertainties are input $\underline{\mathbf{f}}$ and parameter $\underline{\mathbf{s}}$ of the mean model. The best approach to take into 


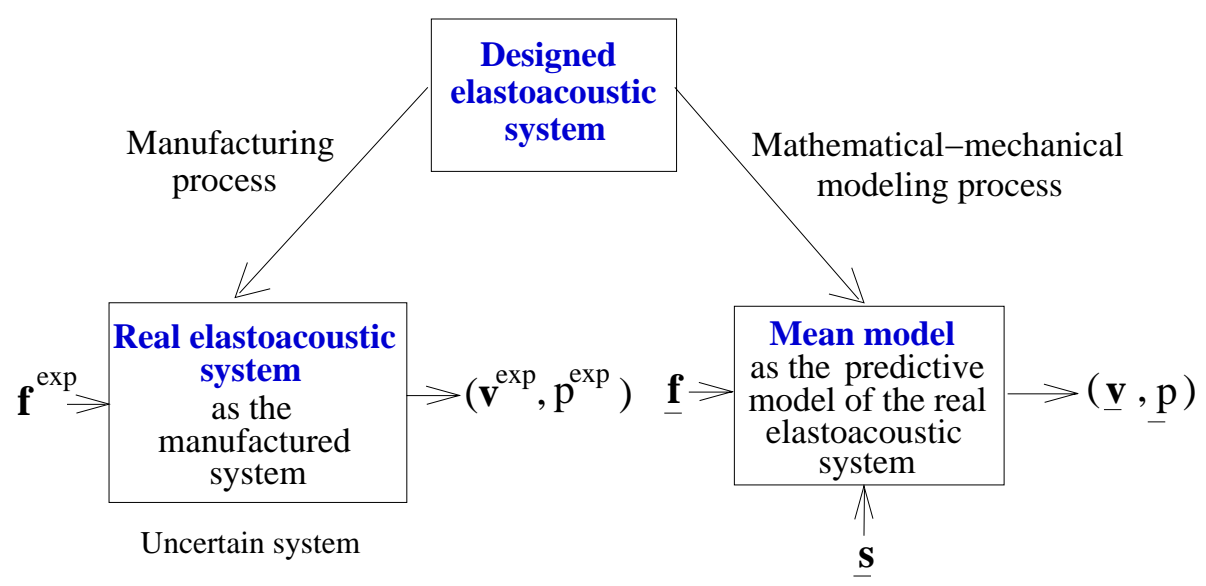

Figure 1: Designed elastoacoustic system, real elastoacoustic system and mean model as the predictive model of the real elastoacoustic system.

account data uncertainties is the parametric probabilistic approach consisting in modeling the data of the mean model by random quantities. The mathematical-mechanical modeling process induces model uncertainties with respect to the designed elastoacoustic system. This type of uncertainties is mainly due to the introduction of simplifications in order to decrease the complexity of the mean model which is constructed. For instance, a slender cylindrical elastic structural element will be modeled by using the beam theory, a thick rectangular plate elastic structural element will be modeled by a thick plate theory, a sound proofing scheme between the structure and the acoustic cavity will be modeled by a wall coustic impedance, the geometry of the acoustic cavity will be simplified, etc. It is clear that the introduction of such simplifications yields a mean model for which all the possible variations of its parameter $\underline{\mathbf{s}}$ do not allow the model uncertainties to be reduced. Model uncertainties have then to be taken into account to improve the predictability of the mean model. As explained above, the parametric probabilistic approach cannot be used. This is the reason why a nonparametric probabilistic approach is proposed. The error between prediction $\left(\underline{\mathbf{v}}^{n}, \underline{p}^{n}\right)$ calculated with the mean model and the response $\left(\mathbf{v}^{\exp }, p^{\exp }\right)$ of the real elastoacoustic system can be measured by $\left(\left\|\mathbf{v}^{\exp }-\underline{\mathbf{v}}^{n}\right\|^{2}+\left\|p^{\exp }-\underline{p}^{n}\right\|^{2}\right)^{1 / 2}$ in which $\|$.$\| denotes appropriate norms. Clearly, the mean model can be considered as a pre-$ dictive model if this error is sufficiently small. In general, due to data uncertainties and model uncertainties, this error is not sufficiently small and has to be reduced by taking into account data uncertainties and model uncertainties.

\section{NONPARAMETRIC PROBABILISTIC APPROACH OF MODEL UNCERTAINTIES}

The concept of the nonparametric probabilistic approach of model uncertainties introduced in [9] is the following (see [10]). Let $\mathbf{s} \mapsto \underline{A}(\mathbf{s})$ be a linear mapping from a space $\mathcal{S}$ into a space $\mathcal{A}$ of linear operators. The space $\mathcal{S}$ represents the set of all possible values of the vectorvalued parameter $\mathbf{s}$ of the boundary value problem (for instance, geometric parameters, elastic properties, boundary conditions, etc). For $\mathbf{s}$ fixed in $\mathcal{S}$, operator $\underline{A}(\mathbf{s})$ represents one operator of the boundary value problem (for instance, the stiffness operator of the structure which is assumed to be symmetric and positive, and in this case, any operator in $\mathcal{A}$ will be symmetric and positive). Let $R_{\text {par }} \subset \mathcal{A}$ be the range of the mapping $\mathbf{S} \mapsto \underline{A}(\mathbf{s})$, i.e. the subset of $\mathcal{A}$ spanned by $\underline{A}(\mathbf{s})$ when $\mathbf{s}$ runs through $\mathcal{S}$. The corresponding operator of the real elastoacoustic system system is $\mathbf{A}^{\exp }$ belonging to $\mathcal{A}$. If $\mathbf{s}=\underline{\mathbf{s}}$ is the nominal value, then $\underline{\mathbb{A}}=\underline{A}(\underline{\mathbf{s}}) \in R_{\text {par }}$ is the operator of the mean model. 


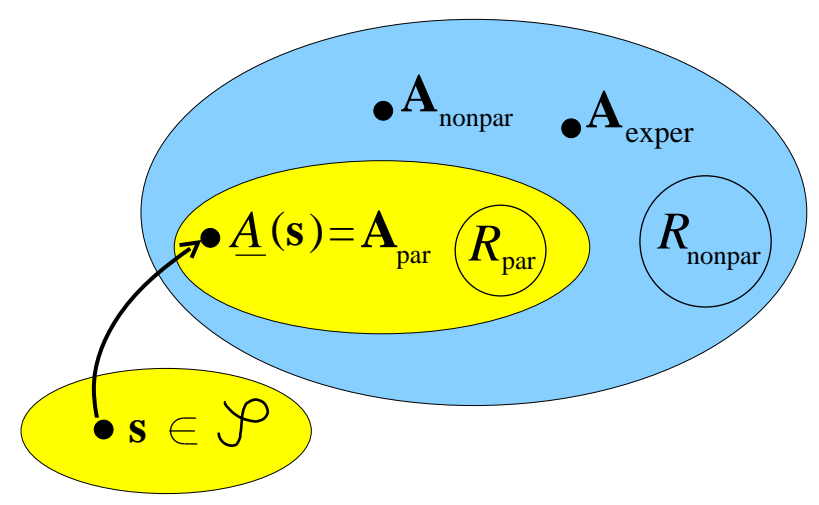

Figure 2: Parametric and nonparametric probabilistic approaches of random uncertainties.

Parametric probabilistic model of the operator. The parametric probabilistic approach for the operator consists in modeling the parameter $\mathbf{s}$ by a vector-valued random variable $\mathbf{S}$ whose probability distribution $P_{\mathbf{S}}(d \mathbf{s})$ has a support which is $\mathcal{S}$. Then the operator $\mathbb{A}$ of the mean model is replaced in the the BVP by the random operator $\mathbf{A}_{\text {par }}$ such that $\mathbf{A}_{\text {par }}=\underline{A}(\mathbf{S})$. The probability distribution $P_{\mathbf{A}_{\mathrm{par}}}$ of the random operator $\mathbf{A}_{\mathrm{par}}$ is $P_{\mathbf{A}_{\mathrm{par}}}=\underline{A}\left(P_{\mathbf{S}}\right)$ and its support is the set $R_{\text {par }} \subset \mathcal{A}$ (see Figure 2). Clearly, the probability $P_{\mathbf{A}_{\mathrm{par}}}$ on $R_{\text {par }}$ allows data uncertainties to be taken into account, but $\mathbf{A}^{\text {exp }}$ may not be in $R_{\text {par }}$ due to model uncertainties.

Nonparametric probabilistic model of the operator. The nonparametric probabilistic approach for the operator consists in replacing the operator $\underline{\mathbb{A}}$ of the mean model by a random operator $\mathbf{A}_{\text {nonpar }}$ whose probability distribution $P_{\mathbf{A}_{\text {nonpar }}}$ has a support which is $R_{\text {nonpar }}=\mathcal{A}$. Since $\mathbf{A}^{\text {exp }}$ belongs to $\mathcal{A}$ and since the support of $P_{\mathbf{A}_{\text {nonpar }}}$ is also $\mathcal{A}$, model uncertainties can be taken into account by the nonparametric approach (see Figure 2). Of course, $P_{\mathbf{A}_{\text {nonpar }}}$ cannot be arbitrary chosen with support $R_{\text {nonpar }}$, but has to be constructed using the available information. Such a methodology has been developed in $[9,10]$ using the information theory.

Methodology. The methodology of the nonparametric probabilistic approach of uncertainties is as follows. (1) Developement of a finite element model of the designed elastoacoustic system. Such a model will be called the mean model (or the nominal model). (2) Construction of a reduced mean model from the mean model. (3) Construction of a stochastic reduced model from the reduced mean model using the nonparametric probabilistic approach which allows the probability distribution of each random generalized matrix to be constructed. (4) Construction and convergence analysis of the stochastic solution.

Experimental identification. The level of uncertainties is controlled by the dispersion parameter of each random matrix introduced in the nonparametric probabilistic approach. In this paper, we present methods for an experimental identification of the dispersions parameters.

\section{STOCHASTIC MODEL OF UNCERTAIN ELASTOACOUSTIC SYSTEMS}

\subsection{Reduced mean model of the elastoacoustic system}

The elastoacoustic system is made up of a viscoelastic structure coupled with an internal acoustic cavity filled with a dissipative acoustic fluid. The usual formulation in "structural displacement" - "acoustic pressure" is used to construct the mean finite element method of the elastoacoustic system (see for instance [6]). Let $\underline{\mathbf{u}}(\omega)$ be the $\mathbb{C}^{n_{s}}$-vector of the $n_{s}$ DOF of the structure and let $\underline{\mathbf{p}}(\omega)$ be the $\mathbb{C}^{n_{f}}$-vector corresponding to the the $n_{f}$ DOF of the acoustic cavity.

Let $\left\{\underline{\varphi}_{1}, \ldots, \underline{\varphi}_{N_{s}}\right\}$ be the $N_{s}$ first structural modes of the structure in vacuo and calculated at 
zero frequency (not including rigid body modes if there exist). Let $\left\{\underline{\Psi}_{1}, \ldots, \underline{\Psi}_{N_{f}}\right\}$ be the $N_{f}$ first acoustic modes of the acoustic cavity with rigid fluid-structure coupling interface (including the constant pressure mode if the acoustic cavity is closed). The reduced mean model is obtained by projection of the mean finite element model on the subspace $V_{N_{s}} \times V_{N_{f}}$ of $\mathbb{R}^{n_{s}} \times \mathbb{R}^{n_{f}}$ in which $V_{N_{s}}$ is spanned by $\left\{\underline{\varphi}_{1}, \ldots, \underline{\varphi}_{N_{s}}\right\}$ and $V_{N_{f}}$ is spanned by $\left\{\underline{\Psi}_{1}, \ldots, \underline{\Psi}_{N_{f}}\right\}$. The reduced mean model can then be written as

$$
\underline{\mathbf{u}}(\omega)=\sum_{\alpha=1}^{N_{s}} \underline{q}_{\alpha}^{s}(\omega) \underline{\varphi}_{\alpha} \quad, \quad \underline{\mathbf{p}}(\omega)=\sum_{\beta=1}^{N_{f}} \underline{q}_{\beta}^{f}(\omega) \underline{\Psi}_{\beta} .
$$

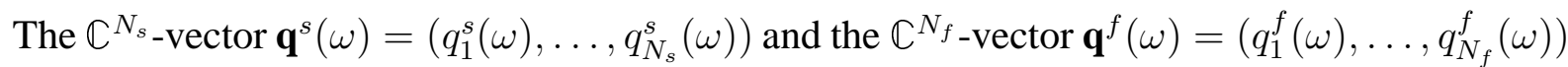
are the solution of the following matrix equation

$$
\left[\begin{array}{cc}
-\omega^{2}\left[\underline{M}_{s}\right]+i \omega\left[\underline{D}_{s}(\omega)\right]+\left[\underline{K}_{s}(\omega)\right] & {[\underline{C}]} \\
\omega^{2}[\underline{C}]^{T} & \left.-\omega^{2}\left[\underline{M}_{f}\right]+i \omega \underline{D}_{f}\right]+\left[\underline{K}_{f}\right]
\end{array}\right]\left[\begin{array}{l}
\underline{\mathbf{q}}^{s}(\omega) \\
\underline{\mathbf{q}}^{f}(\omega)
\end{array}\right]=\left[\begin{array}{l}
\underline{\mathbf{f}}^{s}(\omega) \\
\underline{\mathbf{f}}^{f}(\omega)
\end{array}\right]
$$

in which the $\left(N_{s} \times N_{s}\right)$ real matrices $\left[\underline{M}_{s}\right],\left[\underline{D}_{s}(\omega)\right]$ and $\left[\underline{K}_{s}(\omega)\right]$ are the generalized mass, damping and stiffness matrices of the structure, where the $\left(N_{f} \times N_{f}\right)$ real matrices $\left[\underline{M}_{f},\left[\underline{D}_{f}\right]\right.$ and $\left[\underline{K}_{f}\right]$ are the generalized mass, damping and stiffness matrices of the acoustic and where the rectangular $\left(N_{s} \times N_{f}\right)$ real matrix $[\underline{C}]$ is the generalized vibroacoustic coupling matrix. In Eq. (2) the $\mathbb{C}^{N_{s}}$-vector $\underline{\mathbf{f}}^{s}(\omega)$ and the $\mathbb{C}^{N_{f}}$-vector $\underline{\mathbf{f}}^{f}(\omega)$ are the generalized force vector of the structure and the generalized acoustic source vector of the acoustic cavity respectively.

\subsection{Stochastic reduced model using the nonparametric probabilistic approach}

The principle of construction of the nonparametric probabilistic approach $($ see $[9,10])$ of model uncertainties and data uncertainties in the structure, in the acoustic cavity and for the vibroacoustic coupling consists (1) in modeling the generalized mass $\left[\underline{M}_{s}\right]$, damping $\left[\underline{D}_{s}(\omega)\right]$ and stiffness $\left[\underline{K}_{s}(\omega)\right]$ matrices of the structure by random matrices $\left[\mathbf{M}_{s}\right],\left[\mathbf{D}_{s}(\omega)\right]$ and $\left[\mathbf{K}_{s}(\omega)\right]$ whose dispersion parameters are $\delta_{M_{s}}, \delta_{D_{s}}$ and $\delta_{K_{s}}$ respectively; (2) in modeling the generalized mass $\left[\underline{M}_{f}\right]$, damping $\left[\underline{D}_{f}\right]$ and stiffness $\left[\underline{K}_{f}\right]$ matrices of the acoustic cavity by random matrices $\left[\mathbf{M}_{f}\right],\left[\mathbf{D}_{f}\right]$ and $\left[\mathbf{K}_{f}\right]$ whose dispersion parameters are $\delta_{M_{f}}, \delta_{D_{f}}$ and $\delta_{K_{f}}$ respectively; (3) in modeling the generalized vibroacoustic coupling matrix $[\underline{C}]$ by a random matrix $[\mathbf{C}]$ whose dispersion parameter is $\delta_{C}$. The explicit construction of the probability distribution of these random matrices were performed by using the maximum entropy principle and is given in [9] for random matrices $\left[\mathbf{M}_{s}\right],\left[\mathbf{D}_{s}(\omega)\right],\left[\mathbf{K}_{s}(\omega)\right],\left[\mathbf{M}_{f}\right],\left[\mathbf{D}_{f}\right]$ and $\left[\mathbf{K}_{f}\right]$, and is given in [10] for random matrix $[\mathbf{C}]$. Let $[\mathbf{A}]$ be anyone of these random matrices. In this theory, the probability distribution of such a random matrix $[\mathbf{A}]$ depends only on its mean value $[\underline{A}]=E\{[\mathbf{A}]\}$ in which $E$ is the mathematical expectation and on its dispersion parameter $\delta_{A}$ which is independent of the matrix dimension. In addition, an algebraic representation of random matrix $[\mathbf{A}]$ has been developed and allows independent realizations to be constructed for a stochastic solver based on the Monte Carlo numerical simulation. It should be noted that when $[\mathbf{A}(\omega)]$ is a symmetric positive real-valued matrix depending on $\omega$, then random matrix $[\mathbf{A}(\omega)]$ is written as $[\mathbf{A}(\omega)]=\left[\underline{L}_{A}(\omega)\right]^{T}[\mathbf{G}]\left[\underline{L}_{A}(\omega)\right]$ in which $[\underline{A}(\omega)]=\left[\underline{L}_{A}(\omega)\right]^{T}\left[\underline{L}_{A}(\omega)\right]$ and where the random matrix germ $[\mathbf{G}]$ is independent of $\omega$ and dispersion parameter $\delta_{A}$ must be taken independent of $\omega$. Using such an approach, the stochastic reduced model of the uncertain elastoacoustic system for which the reduced mean model is defined by Eq. (2) is written, for all $\omega$ fixed in the 
frequency band of analysis $B=\left[\omega_{0}, \omega_{1}\right]$ with $0<\omega_{0}<\omega_{1}$, as

$$
\underline{\mathbf{U}}(\omega)=\sum_{\alpha=1}^{N_{s}} \underline{Q}_{\alpha}^{s}(\omega) \underline{\varphi}_{\alpha} \quad, \quad \underline{\mathbf{P}}(\omega)=\sum_{\beta=1}^{N_{f}} \underline{Q}_{\beta}^{f}(\omega) \underline{\Psi}_{\beta},
$$

in which, for $\omega$ fixed in $B$, the $\mathbb{C}^{N_{s}}$-valued random variable $\mathbf{Q}^{s}(\omega)=\left(Q_{1}^{s}(\omega), \ldots, Q_{N_{s}}^{s}(\omega)\right)$ and the $\mathbb{C}^{N_{f}}$-valued random variable $\mathbf{Q}^{f}(\omega)=\left(Q_{1}^{f}(\omega), \ldots, Q_{N_{f}}^{f}(\omega)\right)$ are the solution of the following random matrix equation

$$
\left[\begin{array}{cc}
-\omega^{2}\left[\mathbf{M}_{s}\right]+i \omega\left[\mathbf{D}_{s}(\omega)\right]+\left[\mathbf{K}_{s}(\omega)\right] & {[\mathbf{C}]} \\
\omega^{2}[\mathbf{C}]^{T} & -\omega^{2}\left[\mathbf{M}_{f}\right]+i \omega\left[\mathbf{D}_{f}\right]+\left[\mathbf{K}_{f}\right]
\end{array}\right]\left[\begin{array}{l}
\mathbf{Q}^{s}(\omega) \\
\mathbf{Q}^{f}(\omega)
\end{array}\right]=\left[\begin{array}{l}
\underline{\mathbf{f}}^{s}(\omega) \\
\underline{\mathbf{f}}^{f}(\omega)
\end{array}\right] .
$$

\subsection{Construction and convergence of the stochastic solution}

For all $\omega$ fixed in $B$, it can be proven that the probability model constructed for the random matrices is such that Eq. (4) has a unique second-order solution (see the methodology presented in [9]), i.e., $E\left\{\left\|\mathbf{Q}^{s}(\omega)\right\|^{2}\right\} \leq c_{1}<+\infty$ and $E\left\{\left\|\mathbf{Q}^{f}(\omega)\right\|^{2}\right\} \leq c_{2}<+\infty$. Concerning the stochastic solver, for all $\omega$ fixed in $B$, the stochastic solution of Eq. (4) is constructed by using the Monte Carlo numerical simulation with $m$ independent realizations. Using the usual statistical estimator of the mathematical expectation $E$, the convergence of the stochastic solution with respect to $N_{s}, N_{f}$ and $m$, is studied in constructing the functions $\left(N_{s}, m\right) \mapsto \operatorname{conv}^{s}\left(N_{s}, m\right)$ and $\left(N_{f}, m\right) \mapsto \operatorname{conv}^{f}\left(N_{f}, m\right)$ such that

$$
\begin{aligned}
& \operatorname{conv}^{s}\left(N_{s}, m\right)=\left\{\frac{1}{m} \sum_{k=1}^{m} \int_{B}\left\|\mathbf{U}\left(\omega, \theta_{k}\right)\right\|^{2} d \omega\right\}^{1 / 2}, \\
& \operatorname{conv}^{f}\left(N_{f}, m\right)=\left\{\frac{1}{m} \sum_{k=1}^{m} \int_{B}\left\|\mathbf{P}\left(\omega, \theta_{k}\right)\right\|^{2} d \omega\right\}^{1 / 2},
\end{aligned}
$$

in which $\mathbf{U}\left(\omega, \theta_{1}\right), \ldots, \mathbf{U}\left(\omega, \theta_{m}\right)$ and $\mathbf{P}\left(\omega, \theta_{1}\right), \ldots, \mathbf{P}\left(\omega, \theta_{m}\right)$ are $m$ independent realizations of $\mathbf{U}(\omega)$ and $\mathbf{P}(\omega)$ respectively.

\section{IDENTIFICATION OF THE PROBABILISTIC MODEL PARAMETERS FROM EX- PERIMENTS}

The problem to be solved is related to the experimental identification of the vector-valued dispersion parameter $\boldsymbol{\delta}=\left(\delta_{M_{s}}, \delta_{D_{s}}, \delta_{K_{s}}, \delta_{M_{f}}, \ldots\right)$ introduced in the nonparametric probabilistic approach of data and model uncertainties. Let $\mathbf{Y}(\omega, \boldsymbol{\delta})=\left(Y_{1}(\omega, \boldsymbol{\delta}), \ldots, Y_{\mu}(\omega, \boldsymbol{\delta})\right)$ be the $\mathbb{R}^{\mu}$-valued random variable corresponding to $\mu$ observations of the stochastic reduced model which will be measured for all $\omega$ belonging to frequency band $B$. This vector-valued random variable depends on the vector-valued dispersion parameter $\delta$ which has to be identified using measurements. Since the manufactured systems have a variability induced by the manufacturing process, the corresponding observations of the real system must be modeled by a $\mathbb{R}^{\mu}$-valued random variable $\mathbf{Y}^{\exp }(\omega)=\left(Y_{1}^{\exp }(\omega), \ldots, Y_{\mu}^{\exp }(\omega)\right)$. It is assumed that the measurements are performed for $\nu$ manufactured real systems. Let $\mathbf{y}^{\exp , k}(\omega)=\left(y_{1}^{\exp , k}(\omega), \ldots, y_{\mu}^{\exp , k}(\omega)\right)$ be the $\mathbb{R}^{\mu}$-vector of the $\mu$ measured obervations for manufactured system number $k$. The mean value $\underline{\mathbf{Y}}(\omega, \boldsymbol{\delta})$ of random vector $\mathbf{Y}(\omega, \boldsymbol{\delta})$ and the mean value $E\left\{\mathbf{Y}^{\exp }(\omega)\right\}$ of random vector $\mathbf{Y}^{\exp }(\omega)$ are such that

$$
\underline{\mathbf{Y}}(\omega, \boldsymbol{\delta})=E\{\mathbf{Y}(\omega, \boldsymbol{\delta})\} \quad, \quad E\left\{\mathbf{Y}^{\exp }(\omega)\right\}=\underline{\mathbf{y}}^{\exp }(\omega) \quad, \quad \underline{\mathbf{y}}^{\exp }(\omega)=\frac{1}{\nu} \sum_{k=1}^{\nu} \mathbf{y}^{\exp , k}(\omega)
$$


in which $\underline{\mathbf{y}}^{\exp }(\omega)$ is the experimental mean value. Note that random vector $\mathbf{Y}^{\exp }(\omega)$ is constructed such that its mean value is equal to $\mathbf{y}^{\exp }(\omega)$. Below, we present two methods which can be used to identify the vector-valued dispersion parameter $\delta$ from experiments. The first one will be called the mean-square identification method and can be used for a vector-valued random variable without any difficulties. This method consists in minimizing, in the mean-square sense, the distance between the computed random response and the experimental response. The second one consists in using the maximum likelihood method and can also be used for a vector-valued random variable. Nevertheless, the computational time required by such a method is prohibitive if the vector-valued random variable has a high dimension. Consequently, we will present this method for a real-valued random variable.

\subsection{Mean-square identification method}

Let $\omega \mapsto \mathbf{X}(\omega)=\left(X_{1}(\omega), \ldots, X_{\mu}(\omega)\right)$ be a $\mathbb{R}^{\mu}$-valued second-order stochastic process indexed by frequency band $B$. We introduce the norm $\||\mathbf{X} \|| \mid$ of $\mathbf{X}$ such that

$$
\|\mathbf{X}\|\left\|^{2}=E\left\{\|\mathbf{X}\|_{B}^{2}\right\} \quad, \quad\right\| \mathbf{X}\left\|_{B}^{2}=\int_{B}\right\| \mathbf{X}(\omega) \|^{2} d \omega
$$

in which $\|\mathbf{X}(\omega)\|^{2}=X_{1}(\omega)^{2}+\ldots+X_{\mu}(\omega)^{2}$. The mean square identification of parameter $\delta$ consists in minimizing the cost function $J_{0}(\boldsymbol{\delta})=\left\||| \mathbf{Y}(., \delta)-\mathbf{Y}^{\exp } \mid\right\|^{2}$ with respect to $\boldsymbol{\delta}$. In order to compute this cost function, we can write $\left\|\left|\mathbf{Y}(., \boldsymbol{\delta})-\mathbf{Y}^{\exp }\right|\right\|^{2}=\|\| \mathbf{Y}(., \boldsymbol{\delta})-\underline{\mathbf{Y}}(., \boldsymbol{\delta})-\left(\mathbf{Y}^{\exp }-\right.$ $\left.\underline{\mathbf{y}}^{\exp }\right)+\underline{\mathbf{Y}}(., \boldsymbol{\delta})-\underline{\mathbf{y}}^{\exp }||^{2}$. Since $\underline{\mathbf{Y}}(., \boldsymbol{\delta})-\underline{\mathbf{y}}^{\exp }$ is a deterministic vector and since $\mathbf{Y}(., \boldsymbol{\delta})-\underline{\mathbf{Y}}(., \boldsymbol{\delta})$ and $\mathbf{Y}^{\exp }-\underline{\mathbf{y}}^{\exp }$ are independent and centered vector-valued random variables, we can write

$$
J_{0}(\boldsymbol{\delta})=\left\|\left|\mathbf{Y}(., \boldsymbol{\delta})-\underline{\mathbf{Y}}(., \boldsymbol{\delta})\|\|^{2}+\left\|\mid \mathbf{Y}^{\exp }-\underline{\mathbf{y}}^{\exp }\right\|\left\|^{2}+\right\| \underline{\mathbf{Y}}(., \boldsymbol{\delta})-\underline{\mathbf{y}}^{\exp } \|_{B}^{2} .\right.\right.
$$

In the right-hand side of Eq. (9), the first, the second and the third terms represent the variance of the random response of the stochastic model, the variance of the real system induced by its variability and the bias between the model and the real system, respectively. It should be noted that the second term is independent of $\boldsymbol{\delta}$. Consequently, the cost function $J_{0}(\boldsymbol{\delta})$ can be replaced by a cost function $J_{1}(\boldsymbol{\delta})$ obtained by removing this second term. Consequently, the mean square identification of parameter $\delta$ consists in solving the following optimization problem

$$
\boldsymbol{\delta}^{\text {opt }}=\arg \min _{\boldsymbol{\delta}} J_{1}(\boldsymbol{\delta})
$$

in which the cost function $J_{1}(\boldsymbol{\delta})$ is written as

$$
J_{1}(\boldsymbol{\delta})=\|\mathbf{Y}(., \boldsymbol{\delta})-\underline{\mathbf{Y}}(., \boldsymbol{\delta})\|\left\|^{2}+\right\| \underline{\mathbf{Y}}(., \boldsymbol{\delta})-\underline{\mathbf{y}}^{\exp } \|_{B}^{2}
$$

\subsection{Maximum likelihood method}

For the maximum likelihood method, we introduce the real-valued random variable $Z(\delta)$ for which the $\nu$ independent realizations $z^{\exp , 1}, \ldots, z^{\exp , \nu}$ correspond to the $\nu$ manufactured real systems. Let $p_{Z}(z, \boldsymbol{\delta}) d z$ be the probability distribution on $\mathbb{R}$ of $Z(\boldsymbol{\delta})$ represented by a probability density function $p_{Z}(z, \boldsymbol{\delta})$ which depends on dispersion parameter $\boldsymbol{\delta}$. This random variable is defined by

$$
Z(\boldsymbol{\delta})=\int_{B} \mathrm{~dB}(\omega, \boldsymbol{\delta}) d \omega \quad, \quad \mathrm{dB}(\omega, \boldsymbol{\delta})=10 \log _{10}\left(w_{\mathrm{ref}}^{2} \frac{1}{\mu} \sum_{j=1}^{\mu}\left|Y_{j}(\omega, \boldsymbol{\delta})\right|^{2}\right)
$$


in which $w_{\text {ref }}$ is a constant of normalization. It should be noted that, for all $z$ fixed in $\mathbb{R}$, probability density function $p_{Z}(z, \boldsymbol{\delta})$ can easily be estimated with Eqs. (3) and (4) using the Monte Carlo method and mathematical statistics. For $k=1, \ldots, \nu$, the corresponding realization $z^{\exp , k}$ is written as

$$
z^{\exp , k}=\int_{B} \mathrm{~dB}^{\exp , k}(\omega) d \omega \quad, \quad \mathrm{dB}^{\exp , k}(\omega)=10 \log _{10}\left(w_{\text {ref }}^{2} \frac{1}{\mu} \sum_{j=1}^{\mu}\left|y_{j}^{\exp , k}(\omega)\right|^{2}\right) .
$$

The use of the maximum likelihood method (see [8]) leads us to the following optimization problem

$$
\boldsymbol{\delta}^{\mathrm{opt}}=\arg \max _{\boldsymbol{\delta}} \mathcal{L}(\boldsymbol{\delta})
$$

in which $\mathcal{L}(\boldsymbol{\delta})$ is written as

$$
\mathcal{L}(\boldsymbol{\delta})=\sum_{k=1}^{\nu} \log _{10}\left(p_{Z}\left(z^{\mathrm{exp}, k}, \boldsymbol{\delta}\right)\right)
$$

\section{ANALYZING EXPERIMENTAL CONFIGURATIONS}

\subsection{First experimental configuration: composite sandwich panel coupled with an acous- tic cavity $[1,2]$}

The experimental configuration of the elastoacoustic system is defined in Figure 3. The system is made up of a composite sandwich panel coupled with a closed acoustic cavity constituted of an acoustic box with 5 rigid walls. The designed sandwich panel is constituted of five layers made of four thin carbon-resin unidirectional plies and one high stiffness closed-cell foam core. The geometrical and mechanical parameters for the composite sandwich panel and the acoustic box can be found in $[1,2]$. Eight sandwich panels have been manufactured from the
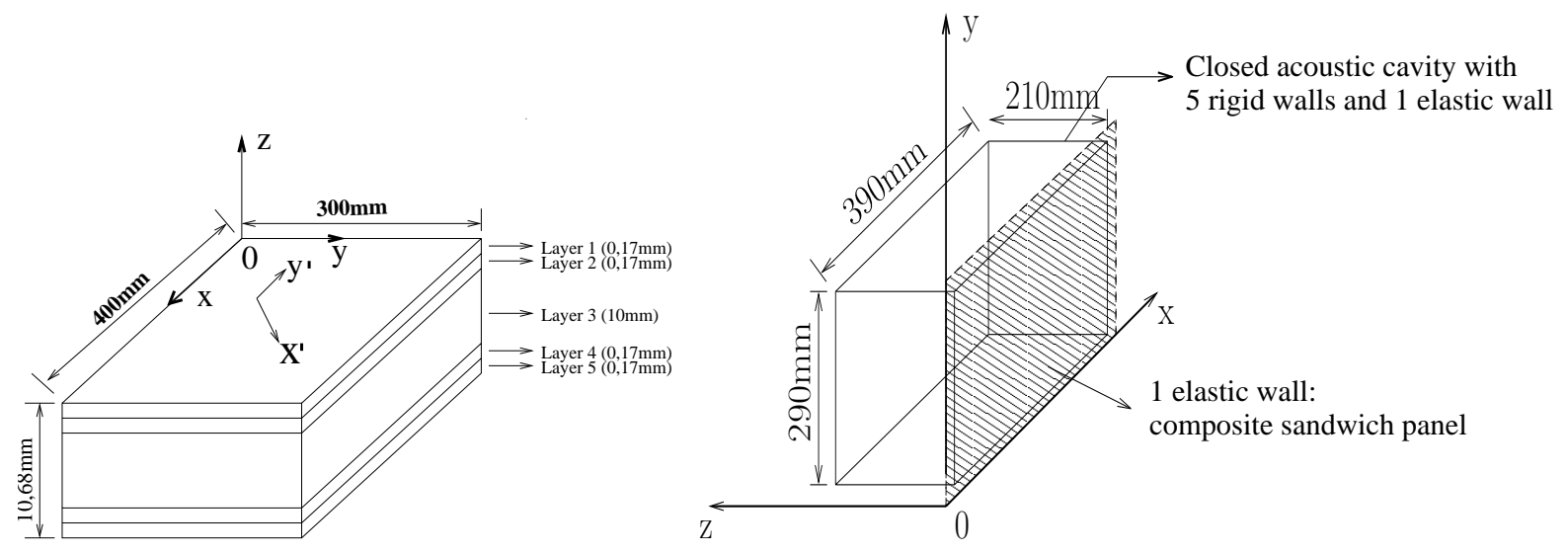

Figure 3: Definition of the composite sandwich panel (left figure). Vibroacoustic system made up of the composite sandwich panel and an acoustic cavity (right figure)

designed sandwich panel using an identical process and the same materials. All the sandwich panels have been baked in the same batch for suppressing the influences of the different baking conditions concerning time and temperature. In addition one acoustic box with 5 rigid walls in the frequency band of analysis has been manufactured. The frequency band of analysis considered is the band $B=[10,4500] \mathrm{Hz}$ corresponding to the model validity of the mean finite 
element model of the vibroacoustic system. The input $z$-force is a point load applied to the point of coordinates $(0.187,0.103,0) \mathrm{m}$. The output $z$-acceleration at the observation point on the panel is the point of coordinates $(0.337,0.272,0) \mathrm{m}$. The mean finite element model of the composite sandwich panel is constituted of $62 \times 46$ four-nodes finite elements for laminated plate bending with orthotropic materials. The finite element model of the acoustic cavity is made up of $60 \times 40 \times 30$ eight-nodes solid acoustic finite elements. Consequently, there are $n_{s}=8556$ structural DOF and $n_{f}=72000$ acoustic DOF. The objective is to analyze the effects of structural uncertainties on the noise produced inside the acoustic cavity by the vibrations of the panel. Consequently, it is assumed that there are uncertainties in the panel but that there are no uncertainties neither in the acoustic cavity nor for the vibroacoustic coupling (this means that $\left.\delta_{M_{f}}=\delta_{D_{f}}=\delta_{K_{f}}=\delta_{C}=0\right)$. Convergence of the stochastic reduced model over frequency band $B$ is obtained for $N_{s}=117$ structural modes, for $N_{f}=630$ acoustic modes and for $m=1300$ realizations. The experimental estimation of the dispersion parameters $\delta_{M_{s}}, \delta_{D_{s}}$ and $\delta_{K_{s}}$ for the composite panels is performed in [1,2] using the method proposed in [10] and yields $\delta_{M_{s}}=0.23, \delta_{D_{s}}=0.43$ and $\delta_{K_{s}}=0.25$. Figures 4 and 5 are related to the experimental

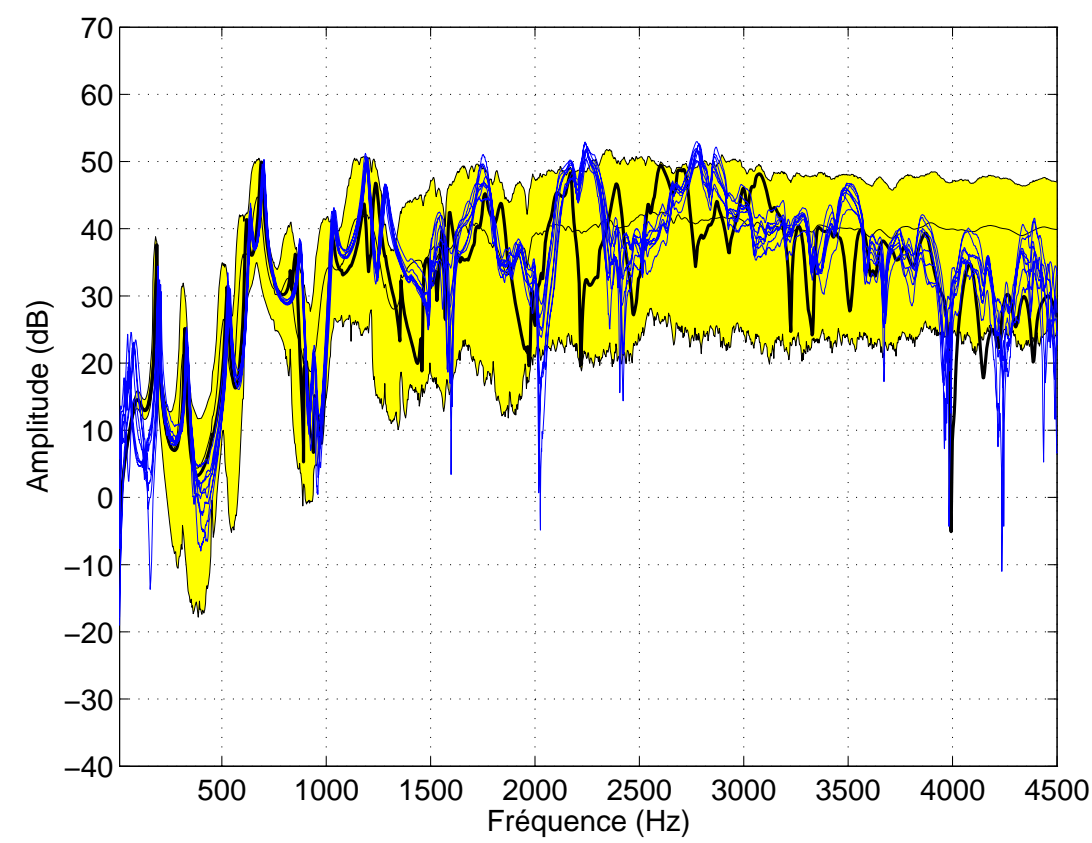

Figure 4: Experimental validation of the confidence region prediction for the random cross FRF between the input force applied to the panel and the transversal acceleration at the observation point on the panel for the vibroacoustic system. Horizontal axis: frequency in Hertz. Vertical axis: $\log _{10}$ of the modulus of the transverse acceleration of the panel. Experimental cross FRF corresponding to the 8 panels ( 8 thin solid lines). Numerical cross FRF calculated with the mean reduced matrix model (thick solid line). Mean value of the random cross FRF calculated with the non parametric probabilistic model (thin solid line). Confidence region of the random cross FRF calculated with the non parametric probabilistic model (grey region).

validation of the confidence region prediction for the random cross frequency response functions (FRF) relative to the panel acceleration and to the acoustic pressure, and corresponding to a probability level of 0.96 (grey region). Figure 4 shows a good experimental comparison for the confidence region calculated with the stochastic reduced model. It should be noted that the confidence region is relatively narrow in the low-frequency (LF) band $[10,1200] \mathrm{Hz}$ and is broad in the medium-frequency (MF) band $[1200,4500] \mathrm{Hz}$. These results show that the mean model is robust with respect to data and model uncertainties of the structure in the LF band but 


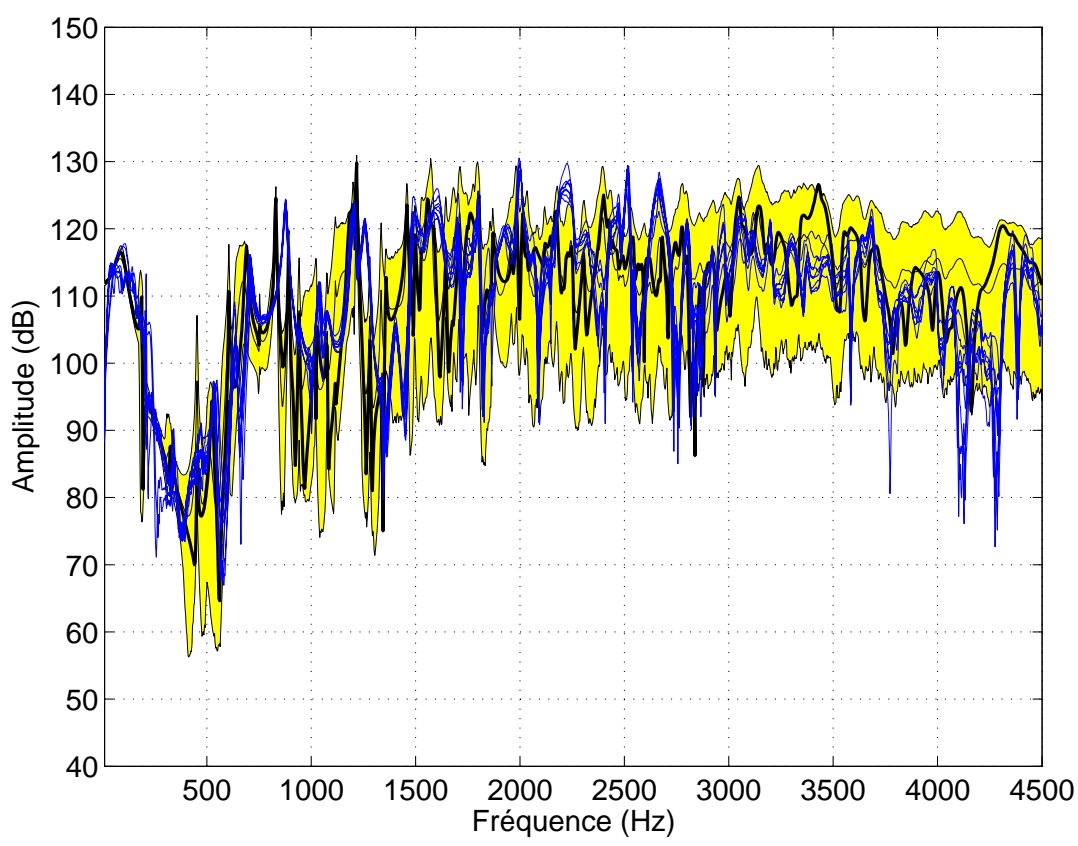

Figure 5: Experimental validation of the confidence region prediction for the random cross FRF between the input force applied to the panel and the acoustic pressure at the observation point in the acoustic cavity for the vibroacoustic system. Horizontal axis: frequency in Hertz. Vertical axis: $\log _{10}$ of the modulus of the acoustic pressure inside the acoustic cavity. Experimental cross FRF corresponding to the 8 panels ( 8 thin solid lines). Numerical cross FRF calculated with the updated mean reduced matrix model (thick solid line). Mean value of the random cross FRF calculated with the non parametric probabilistic model (thin solid line). Confidence region of the random cross FRF calculated with the non parametric probabilistic model (grey region).

is less robust in the MF band. Such a result can also be viewed in comparing the response of the reduced mean model with the mean value of the random response of the stochastic reduced model. In the LF band the mean value of the random response is closed to the response of the mean model while large differences can occur in the MF band. Similarly, Figure 5 shows a good experimental comparison for the confidence region of the internal noise. It can be seen that the mean model is robust with respect to data and model uncertainties of the structure not only in the LF band $[10,1200] \mathrm{Hz}$ but also in the low part $[1200,3000] \mathrm{Hz}$ of the MF band while the robustness decreases in the high part $[3000,4500] \mathrm{Hz}$ of the MF band. It can be conclude that the propagation of uncertainties from the structure into the acoustic cavity is weak in the frequency band $[10,3000] \mathrm{Hz}$ (LF band and low part of the MF band) and increases in the frequency band $[3000,4500] \mathrm{Hz}$ (high part of the MF band).

\subsection{Second experimental validation: vibroacoustics of cars [3,4]}

We present an experimental validation of the numerical prediction of internal noise in a car due to engine excitation applied to the engine supports (booming noise). The mean finite element model is shown in Figure 6. The structure is modeled with $n_{s}=978,733$ DOF of displacement and the acoustic cavity with $n_{f}=8,139 \mathrm{DOF}$ of pressure. The frequency band of analysis $B=[33,200] \mathrm{Hz}$ corresponding to $[1000,6000] \mathrm{rpm}$ (engine rotation per minute). Convergence of the stochastic reduced model over frequency band $B$ is obtained for $N_{s}=1722$ structural modes, for $N_{f}=57$ acoustic modes and for $m=600$ realizations. The experimental identification of the dispersion parameters are performed in three steps as follows (see $[3,4]$ ). For the first step, acoustic pressure measurements have been performed inside the acoustic 

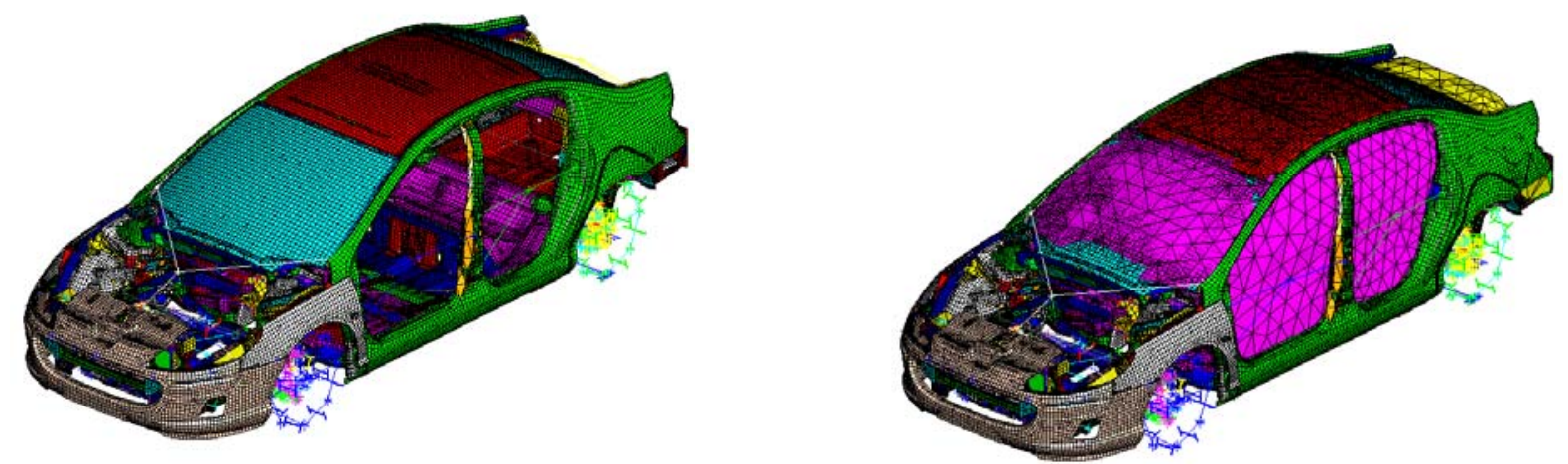

Figure 6: Finite element mesh of the structure: 978,733 DOF of displacement (left figure). Finite element mesh of the acoustic cavity: 8,139 DOF of pressure (right figure)

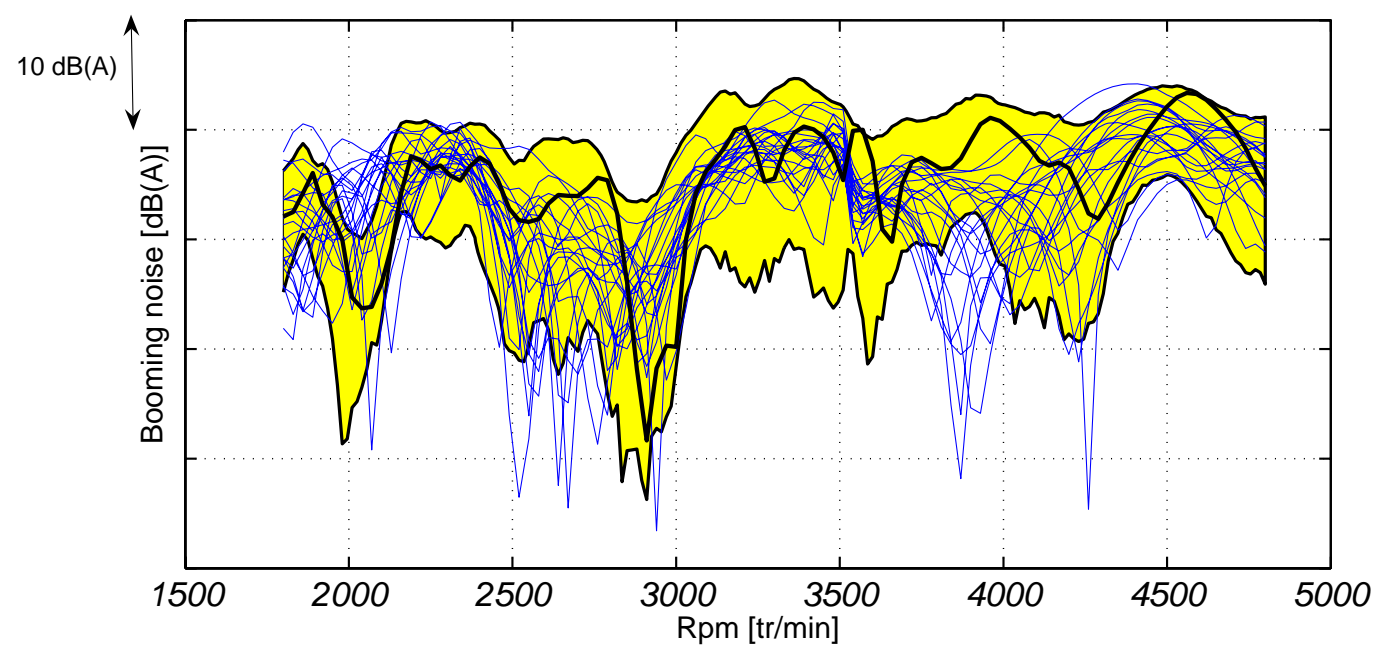

Figure 7: Experimental validation of the confidence region prediction for the random cross FRF between the input force applied to engine supports and the acoustic pressure at an observation point in the acoustic cavity for the vibroacoustic system. Horizontal axis: tr/min. Vertical axis: modulus of the acoustic pressure in dBA. 22 experimental measurements for 22 cars of the same type (22 thin solid lines). Numerical prediction of the mean reduced matrix model (thick solid line). Confidence region of the internal noise predicted with the non parametric probabilistic model and for probability level 0.95. (grey region).

cavity for a given acoustic source inside the cavity. Then the maximum likelihood method described in Section 5.2 has been used taking $\delta_{M_{f}}=\delta_{D_{f}}=\delta_{K_{f}}$, where $Y_{j}(\omega, \boldsymbol{\delta})=P_{\ell_{j}}(\omega)$ in which $P_{\ell_{1}}(\omega), \ldots, P_{\ell_{\mu}}(\omega)$ are the observed acoustic pressures which are measured inside the cavity, with $w_{\text {ref }}=1 / P_{\text {ref }}$ in which $P_{\text {ref }}$ is a reference pressure. For the second step, structural acceleration measurements have been performed in the structure for driven forces applied to the engine supports. Then the mean-square identification method described in Section 5.1 has been used with $Y_{j}(\omega, \boldsymbol{\delta})=\log _{10}\left(w_{j}\left|U_{\ell_{j}}(\omega)\right|\right)$ in which $U_{\ell_{1}}(\omega), \ldots, U_{\ell_{\mu}}(\omega)$ are the observed displacements which are measured and where $w_{1}, \ldots, w_{\mu}$ are normalization constants such that $0<w_{j} \leq 1$. In a third step, dispersion parameter $\delta_{C}$ of the vibroacoustic coupling operator has been fixed at a given value. Figure 7 displays the experimental validation of the numerical prediction of internal noise due to engine excitation with structure, vibroacoustic coupling and acoustic cavity uncertainties. Taking into account the complexity of the vibroacoustic system, there is a good experimental validation of the stochastic elastoacoustic model with model and 
data uncertainties. The variability of the manufactured real systems is due to the process and to the extra options. The propagation of uncertainties is significant in the frequency band of analysis.

\section{CONCLUSIONS}

Data and model uncertainties can be taken into account in computational elastoacoustics by using the nonparametric probabilistic approach. Methodologies are proposed to perform an experimental identification of the dispersion parameters controlling the level of uncertainties. This approach has been validated for a simple and for a complex elastoacoustic system.

\section{REFERENCES}

[1] C. Chen, Vibration et vibroacoustique des panneaux composites sandwich en présence d'incertitudes - Expérimentation et validation du modèle. Thesis of the university of Marne la Vallée, Paris, France, 2006.

[2] C. Chen, D. Duhamel, C. Soize, Probabilistic approach for model and data uncertainties and its experimental identification in structural dynamics: case of composite sandwich panels. Journal of Sound and vibration, 294, 64-81, 2006.

[3] J.-F. Durand, Modélisation de véhicules automobiles en vibroacoustique numérique avec incertitudes et validation expérimentale. Thesis of the University of Marne la Vallée, Paris, France, 2007.

[4] J.-F. Durand, L. Gagliardini, C. Soize, Nonparametric modeling of the variability of vehicle vibroacoustic behavior, SAE Noise and Vibration Conference and Exhibition, Traverse City, Michigan, USA, May 16-19, 2005.

[5] J. Lighthill, Waves in Fluids. Cambridge University Press, MA, 1978.

[6] R. Ohayon, C. Soize, Structural Acoustics and Vibration. Academic Press, San Diego, 1998.

[7] A. D. Pierce, Acoustics: An Introduction to its Physical Principles and Applications. Acoust. Soc. Am. Publications on Acoustics, Woodbury, NY, USA, 1989 (originally published in 1981, McGraw-Hill, New York).

[8] R .J. Serfling, Approximation Theorems of Mathematical Statistics, John Wiley \& Sons, 1980.

[9] C. Soize, Maximum entropy approach for modeling random uncertainties in transient elastodynamics, Journal of the Acoustical Society of America, 109(5), 1979-1996, 2001.

[10] C. Soize, Random matrix theory for modeling uncertainties in computational mechanics. Computer Methods in Applied Mechanics and Engineering, 194(12-16), 1333-1366, 2005. 